\title{
Solar plasma generated by sungrazing comets
}

\author{
F. S. Ibodov ${ }^{1}$ and S. Ibadov ${ }^{1,2}$ \\ ${ }^{1}$ Sternberg Astronomical Institute, Moscow State University, Moscow \\ email: mshtf@sai.msu.ru, firuz@pochta.ru \\ ${ }^{2}$ Institute of Astrophysics, Tajik Academy of Sciences, Dushanbe \\ email: ibadovsu@yandex.ru
}

\begin{abstract}
It is analytically shown that passages of comets near the Sun's surface with velocities more than $600 \mathrm{~km} / \mathrm{s}$ is accompanied by aerodynamic crushing of their nuclei within the solar chromosphere and transversal expansion of the crushed matter. The deceleration of the flattened hypervelocity body within the solar photosphere has sharply impulsive and strongly explosive character. The specific energy release in the explosion zone near the solar surface 10100 thousand times exceeds the evaporation heat of the nucleus material, so that the process is accompanied by generation of high-temperature plasma and non-stationary explosive phenomena around the photosphere. Spectral observations of these phenomena by SOHO and SDO type space observatories with high spatial and temporal resolutions are of interest for the plasma astrophysics as well as the physics of solar flares.
\end{abstract}

Keywords. Sun: general, sungrazing comets, solar impact plasma, solar photospheric flares

\section{Introduction}

Progress in discoveries of sungrazing comets (COSPAR 1998, ESA 2008, NASA 2008) achieved owing to coronagraphic observations of circumsolar region by such space missions as SOLWIND, SMM (Solar Maximum Mission) and SOHO (Solar and Heliospheric Observatory) as well as results of theoretical investigations on the origin and dynamics of sungrazing comets, on the basis of many-body celestial mechanics (Bailey et al. 1992), made actual the study of physical evolution of comet nuclei within the solar dense atmosphere, i.e. taking into account aerodynamic effects.

The physical evolution of comet nuclei in the field of solar photon radiation is sufficiently studied. In particular, it is established that the decrease of a radius of comet nuclei passing near the photosphere of the Sun is limited by a value no more than 15-20 meters, while aerodynamic effects are not considered at all, i.e. vacuum approximation is used (e.g. Weissman 1983, MacQueen \& St. Cyr 1991).

Meantime, parabolic velocity of comets near the solar surface is equal to $617 \mathrm{~km} / \mathrm{s}$, while the density of the solar atmosphere within the chromosphere considerably exceeds $10^{-15} \mathrm{~g} / \mathrm{cm}^{3}$ being of the order of $10^{-7} \mathrm{~g} / \mathrm{cm}^{3}$ in the photosphere. In such situation aerodynamic pressure on the comet nuclei considerably exceeds tidal disruption of the nuclei (Grigoryan et al. 1997).

Besides, the specific kinetic energy of comet nuclei in the inner heliosphere, especially near the surface of the Sun, essentially exceeds the evaporation/sublimation energy of comet nuclei material $10^{10}-10^{11} \mathrm{erg} / \mathrm{g}$, so that plasma phenomena in the region should be taken into account (Ibodov et al. 2006).

In the present paper some properties of plasma produced by sungrazing comet nuclei near the solar surface are considered and connection of the process with solar photospheric flares is marked. 


\section{Solar plasma generated by sungrazing comets}

Calculations show that comet nuclei moving through the chromosphere with orbital velocities more than $600 \mathrm{~km} / \mathrm{s}$ will be aerodynamically destructed. Besides, the intensity of energy flux related to the flow of bombarding atmospheric particles to the comet nucleus surface, $\mathrm{J}_{a}$, essentially exceeds the intensity of energy flux to the comet nucleus due to thermal photon radiation from the solar photosphere, $J_{\lambda}$. It means that near the photosphere comet nuclei with practically all radii will be destroyed/fragmented and evaporated, in principle, due to aerodynamic effect (Ibadov et al. 2007).

Moreover, the aerodynamic pressure gradient on the frontal surface of the comet nucleus leads to transversal expansion of the fragmented comet nucleus matter. Due to this process an aerodynamic deceleration of hypervelocity matter acquires impulse character. Namely, calculations show that the length of the sharp deceleration of flattened comet nucleus matter will be around $0.7 \mathrm{H}$, where $\mathrm{H}=200 \mathrm{~km}$ is the characteristic altitude scale of the solar photosphere (Ibadov et al. 2008).

The atmospheric mass within sharp deceleration layer is of the order of comet nucleus mass. The situation in this region is similar to that for high velocity collision of two dust particles (Ibadov 1992, Ibadov 1996), so that the initial temperature of the impact region in the sub-photosphere layer can be estimated as

$$
T=\frac{A m_{p} V^{2}}{12 k\left(1+z+2 x_{1} / 3\right)} .
$$

Here $\mathrm{A}$ is the mean atomic number for the falling comet nucleus material and matter of solar photosphere, $\mathrm{m}_{p}$ is the proton mass, $\mathrm{k}$ is the Boltzmann constant, $\mathrm{z}$ is the mean multiplicity of charge of plasma ions produced during thermalization of the kinetic energy of comet nucleus in the sub-photosphere layer, $\mathrm{x}_{1}$ is the mean relative ionization potential.

Using (2.1) with realistic values of $\mathrm{A}=20, \mathrm{z}=5, \mathrm{x}_{1}=3$ we obtain for the initial temperature of impact produced solar plasma $T=10^{7} \mathrm{~K}$. This value is considerably more than the temperature of the solar corona plasma, so that the impact generated sub-photosphere explosive process may be observed as a solar photospheric flare.

\section{Conclusions}

Passages of nuclei of sungrazing comets through the solar chromosphere are accompanied by aerodynamic destruction and transversal expansion of the fragmented hypervelocity mass. A sharp aerodynamic deceleration of the nucleus having flattened form in the sub-photosphere layer leads to impulse thermalization of the kinetic energy of the nucleus and generation of solar impact plasma with initial temperature more than the temperature of the solar corona plasma.

Space spectral observations of solar impact plasma / solar photospheric flare, generated by sungrazing comets, with high spatial and temporal resolutions using space observatories like SDO (Solar Dynamics Observatory) are of interest for the physics of solar flares and plasma astrophysics.

\section{Acknowledgements}

The authors are grateful to the IAU Symposium No. 274 SOC/LOC for a travel grant. The hospitality of SAI MSU and DIC MSU as well as the assistance of Dr. Konstantin V. Bychkov and Oleg V. Egorov are acknowledged. 


\section{References}

Bailey, M. E., Chambers, J. E., \& Hahn, G. 1992, A\&A A, 257, 315

COSPAR Inform. Bull. 1998, 142, 22

ESA 2008, ESA-Space Sci., "SOHO Discovers its $1500^{\text {th }}$ Comet", http://www.esa.int/esaSC/, http://sohowww.nascom.nasa.gov/hotshots/2008 $0623 /$

Grigoryan, S. S., Ibodov, F. S., \& Ibadov, S. 1997, Dokl. Akad. Nauk, 354, 187, Engl. Transl.: Phys.-Dokl., 42, 262

Grigoryan, S. S., Ibadov, S., \& Ibodov, F. S. 1998, Cometary Nuclei in Space and Time, IAU Colloquium No. 168 Abstracts, Nanjing, China, 13

Grigoryan, S. S., Ibadov, S., \& Ibodov, F. S. 2000, Dokl. Akad. Nauk, 374, 40 Engl. Transl.:Dokl.-Phys., 45, 463

Ibadov, S. 1992, AZh, 69, 737

Ibadov S. 1996, Physical Processes in Comets and Related Objects, Moscow, Cosmosinform Publishing Company

Ibadov, S., Ibodov, F. S., \& Grigoryan, S. S. 2007, Star-Disk Interaction in Young Stars, Proc. IAU Symp. No. 243, Grenoble, France, www.iaus243.org

Ibadov, S., Ibodov, F. S., \& Grigorian, S. S. 2008, Universal Heliophysical Processes, Proc. IAU Symp. No. 257, N. Gopalswamy \& D. F. Webb, eds., Cambridge University Press, 341 http://iau257.uoi.gr/

Ibodov, F. S., Grigoryan, S. S., \& Ibadov, S. 2006, $36^{\text {th }}$ COSPAR Scientific Assembly Abstracts, Beijing, China, B0.4-0068-06 www.cospar-assembly.org

MacQueen, R. M. \& St. Cyr, O. C. 1991, Icarus, 91, 96

NASA 2008, http://sohowww.nascom.nasa.gov/hotshots/2008_06_23/

Weissman, P. R. 1983, Icarus, 55, 448 